closest reasonable medical facility or to the patient's home country.

Problems related to long-distance air-medical transport include: 1) The pilot's duty-time restrictions combined with the demand for the quickest possible transfer of the patient to a receiving hospital; and 2) Long-range, wide-body aircraft are too expensive to use for air-medical transport.

Solution: A functional world-wide service network created by service providers in different geographical areas should be established by which a coordinated, unbroken logistic chain of air-medical transport service providers would use several air-ambulance aircraft in combination with commercial intercontinental airline services.

Benefits: Expanded world-wide service program would be available for patients wherever the need arises. This would provide cost-effective, reliable, and coordinated repatriation of the patients under supervision of airmedical professionals.

Key words: air-medical transport; network; cost-effective transportation; transportation

\section{Prehospital Thrombolysis: The Medi-Heli Approach}

Löfstedt TJ, Reitala J, Kinnunen AA

EMA Group Ltd (Emergency Medical Assistance), Helsinki, Finland

\section{Background}

I.1. The EMA Group Ltd (Emergency Medical Assistance) has performed 24-hour emergency medical evaluations, assistance, and evacua tion/ repatriation services since 1989.

I.2. EMA evaluates/assists 1,000 cases and arranges 300 medical evacuations/patient transfers annually.

I.3. Since 1992, EMA has provided the physician for Medi-Heli, the Southern Finland Heli II. Outline copter Emergency Medical Service (HEMS)

II.1.Medi-Heli performs 1,700 scene-response missions annually.

II.2. The Unit sees and treats $90-100$ patients with acute myocardial infarction (AMI) every year.

II.3. Of these patients, 30-35 receive prehospital thrombolysis within 90 minutes from onset of first chest pain symptoms.

II.4. Normally, the patient is transported by ground ambulance after the HEMS crew intervention.

III. Objectives and Conclusions

III.1. The importance of thrombolysis as soon as possi ble after onset of the first symptoms is empha sized.

III.2. The HEMS crew can perform diagnosis of AMI on scene.

III.3. The HEMS crew, in addition to other treatment, can start and perform prehospital thrombolysis.

III.4. The HEMS crew can both treat and stabilize the
AMI patient on scene prior to the transportation by ground ambulance.

Key words: air medical; ambulance; chest pain; emergency medical care; emergency medical evaluations; helicopter; myocardial infarction; prehospital; thrombolysis; transportation

\section{Brain Injury in Persons Exposed to Chronic Intoxication by Neurotropic Poisons}

Rabinovich SS, Ivochkin AM, Akentyev NS, Plushch IV Siberian Center for Disaster Medicine, Novosibirsk, Russia

The consequences of chronic intoxication with neurotropic poisons on brain was studied in 138 patients. All of them were poisoned with ethanol or its surrogates. Mainly (87\%), these were men at an age from 46 to 78 years. The age of the women ranged from 38 to 69 years.

All of the patients examined had suffered a severe brain injury which demonstrated some peculiarities connected with brain morpho-functional changes caused by chronic ethanol poisoning. These changes were manifested as: 1) chronic poisoning with ethanol brought about the development of brain atrophy in $43 \%$ of the cases (59 patients). As a result, the ventricular spaces had become enlarged and chronic subdural hematomas had formed. Furthermore, sharp and subsharp subdural hematomas run with 3-5 days gaps in $20 \%$ cases ( 28 patients); 2) Internal hydrocephaly ex vacuo was identified with magnetic resonance computed tomography in major cases studied. Atrophy of visceral brain structures was revealed clinically as the following psychoorganic syndromes: Korsakov's dementia, 32\%; a syndrome with amnesia and abulia, 28\%; epileptic fits, 22\%; 3) Brain blood flow disorders registered by transcranial doppler monitoring as a total vasospasm were observed in $78 \%$ of the cases.

Nimodipine administration for $7-10$ days reduced these phenomena. Thereby, chronic intoxication by neurotropic poisons such as an ethanol, changes in brain pathomorphology and clinical pictures must be considered when rendering a care to this category of damaged beings during evacuation stages.

Key words: brain atrophy; brain injury; cerebral blood flow; chronic intoxication; clinical findings; neurotropic poisons; nimodipine; pathomorphology; subdural hematoma

\section{Evaluation of Japanese Emergency Systems for} Out-of-Hospital Cardiopulmonary Arrest Saitob D, Ishibara $S$, Kaneko $N$, Yanagawa $Y$, Fukuzuka K, Ito T, Kiyozumi T, Sakamoto T, Ohno H, Okada Y

Department of Traumatology and Critical Care Medicine, Department of Hygiene, National Defense Medical College, Tokorozawa, Japan 
Objective: The aim of this study was to evaluate the effectiveness of the Japanese emergency system for outof-hospital cardiopulmonary arrest (oh-CPA).

Background: Sudden CPA patients in Tokorozawa City and the surrounding cities (population of approximately one million) are transported to the hospital by the Japanese Emergency Medical Service (EMS). In the past, Japanese EMS personnel were permitted to perform only bag-valve-mask ventilation and external cardiac compressions for CPA patients. However, since 1991, specially trained emergency medical technicians (EMT) have used defibrillators and intubation devices (except for endotracheal tubes).

Patients and Methods: 1,039 CPA cases were studied. Prognostic factors influencing outcomes from CPA were evaluated using multivariate analysis (quantification theory type); these variables included etiology, age, gender, witnessed arrest, bystander CPR, crew of EMS (EMT or not), time interval from collapse to arrival, and arrival status (CPA or not).

Results: Spontaneous circulation returned in 393/1039 patients $(37.8 \%)$, and $263(25.3 \%)$ were admitted to the hospital wards. Forty-eight (4.6\%) survived, and 13 (1.3\%) recovered fully. Five full recovery cases were resuscitated by an EMT, and four of them returned to spontaneous circulation following defibrillation during ambulance transport. Influential factors for survival were arrival status, time, and etiology.

Conclusion: Further improvement of the Japanese EMS system is needed. Continued EMT education will be necessary to accomplish the goal.

Key words: airway management; cardiopulmonary arrest; defibrillation; emergency medical technicians (EMTs); intubation; outcome; prehospital; resuscitation; survival

\section{ABSTRACTS OF INVITED AND SCIENTIFIC PAPERS FREE Papers Poster Presentations}

\section{Organophosphate Insecticides Poisoning- Causes of Death Bonitenko UU, Bonitenko EU, Tonkopi DV, Aksenov IV All Russian Center of Ecological Medicine, Military-Medical Academy, Saint Petersburg, Russia}

Introduction: Remarkable improvement in the results of the management of acute peroral poisonings with organophosphate insecticides (OPI) have been attained. Despite these gains, the problem has not resolved completely. A mortality rate of $25-30 \%$ still exists.

Subjects: The causes of death in the group of patients admitted with acute peroral poisoning of OPI were studied. The data were abstracted from clinical observations and results of medical forensic examinations.

Results: The causes of death in the group of 87 patients who died of acute OPI poisoning were investigated. Of the total number of deaths, $39.1 \%$ occurred during the toxicogenic phase and $60.9 \%$. during the somatogenic phase.

It should be emphasized that within the first $12 \mathrm{~h}$, the main causes of death were due to coma with brain oedema and respiratory center paralysis and exotoxic shock ( $90 \%$ of all deaths). In the late toxicogenic phase $(12-48 \mathrm{~h})$, the main cause of death was exotoxic shock $(65 \%)$. In the early somatogenic phase (3-6 days) the main cause of death was related to complications due to infection, mostly pulmonary (48.8\%), and hemodynamic failure. The forms of hemodynamic failure were acute left ventricular failure, secondary somatogenic collapse, and dysrythmias despite treatment with potassium. There were no significant differences between the clinician's and pathologist's decisions regarding the cause of death.
Conclusion: These data provide directions to improve the methods of intensive care for acute OPI poisoning.

Key words: dysrhythmias; hemodynamic failure; intensive care; left ventricular failure; organophosphate poisoning; phases of care; secondary infection; shock

\section{Do Medical Curricula \\ Produce Emergency Preparedness? \\ Mattila $M A K$ \\ University of Kuopio, Kuopio, Finland}

Introduction: Every qualified medical doctor is expected to act effectively and logically in a sudden emergency situation. Ideally, this preparedness should be reached gradually during the medical curriculum in the university before qualification. According to anecdotal information, many young medical doctors have uncertain feelings about their abilities when they start their work as general practitioner or have their first night duty in a minor hospital. Because there are no documented data of preparedness or skills achieved with guided training, we studied the medical students in Finland immediately before they completed their studies.

Methods: We personally submitted a standard questionairre to all medical students of the five medical faculties and asked questions concerning their theoretical knowledge, expertise, and readiness to deal with the most usual emergencies.

Results: The answers indicate that the theoretical knowledge included in lectures is estimated to be sufficient, but that the practical training of emergency procedures is $\mathrm{lim}^{-}$ ited to only a few if any, occasions. Their estimation of 